\title{
Landscape equivalent of the shoving model
}

\author{
Jeppe C. Dyre and Niels Boye Olsen \\ Department of Mathematics and Physics (IMFUFA), Roskilde University, P.O. Box 260, DK-4000 Roskilde, Denmark
}

(Received 5 November 2002; revised manuscript received 7 May 2003; published 30 April 2004)

\begin{abstract}
It is shown that the shoving model expression for the average relaxation time of viscous liquids, according to which the activation energy is proportional to the instantaneous shear modulus, follows largely from a classical "landscape" estimation of barrier heights from curvature at energy minima. Although the activation energy in this reasoning involves both instantaneous bulk and shear moduli, the bulk modulus contributes less than $8 \%$ to the temperature dependence of the activation energy. This reflects the fact that the physics of the two models are closely related.
\end{abstract}

DOI: 10.1103/PhysRevE.69.042501

PACS number(s): 64.70.Pf, 62.20.Dc, 62.60.+v

The physics of highly viscous liquids approaching the calorimetric glass transition continue to attract attention [1-9]. A major mystery surrounding these liquids is their non-Arrhenius behavior. If $\tau$ is the average relaxation time and $\tau_{0}$ the microscopic time (of the order of $10^{-13} \mathrm{~s}$ ), the temperature-dependent activation (free) energy $\Delta E(T)$ is defined [10-12] by

$$
\tau(T)=\tau_{0} \exp \left(\frac{\Delta E(T)}{k_{B} T}\right) .
$$

Only few viscous liquids show Arrhenius temperature dependence of the average relaxation time, i.e., have constant $\Delta E(T)$. Most viscous liquids have an activation energy which increases upon cooling.

The shoving model [13] starts from the standard picture of a viscous liquid: At high viscosity almost all molecular motion goes into vibrations around potential energy minima. Only rarely do rearrangements take place which move molecules from one to another minimum. This view was formulated already by Kauzmann in his famous 1948 review [14], and it was the starting point of Goldstein's "potential energy picture" [15] which was later confirmed by computer simulations [16]. On the short-time scale of the barrier transition-expected to last just a few picoseconds- the surrounding liquid behaves as a solid with bulk and shear moduli equal to the instantaneous (i.e., high frequency) bulk and shear moduli. Just as in free volume theories, the shoving model assumes that molecular rearrangements take place when a thermal fluctuation leads to extra space being created locally. One may think of the surroundings as being shoved aside to make the rearrangement possible (although this cause-effect reasoning violates time-reversal symmetry). If there is spherical symmetry, the surroundings are subject to a pure shear displacement $[7,13,17]$. Thus the work done is proportional to the instantaneous shear modulus $G_{\infty}$ and one finds [13] (where $V_{c}$ is by assumption temperature independent)

$$
\Delta E(T)=G_{\infty}(T) V_{c} .
$$

This expression [18] fits data for the non-Arrhenius behavior of several molecular liquids [13]; in combination with the Tool-Narayanaswami formalism the shoving model has been applied also to structural (i.e., nonlinear) relaxations [19]. It is the high-frequency shear modulus which appears in Eq. (2) because the transition is fast (compare, e.g., the analogous appearance of short-time friction constant in the GroteHynes theory [20]).

The basic assumptions of the shoving model may be summarized as follows [7]:

(a) The main contribution to the activation energy is elastic energy.

(b) The elastic energy is located in the surroundings of the rearranging molecules.

(c) The elastic energy is shear energy, i.e., not associated with any density change.

The purpose of this paper is to give an alternative justification of Eq. (2) and discuss the interrelation between the two approaches.

We start by reviewing a classical argument estimating the height of the barrier between two potential energy minima from the curvature around the minima. This argument is used, e.g., in the Marcus theory for electron transfer reactions [21,22]. In 1987 Hall and Wolynes applied this reasoning in their theory of free energy barriers in glasses [23-25]. Consider first the one-dimensional situation with two minima separated the distance $a$ (Fig. 1). The thin curves give the potential estimated by second-order expansions around the minima. The figures show two situations where the minima are the same distance apart, but the potential is scaled going from (a) to (b). In both cases the barriers estimated from the parabolic approximation are considerably larger than the actual barriers. The estimated and actual barriers are proportional, however, which is enough for the arguments of this paper. To express the estimated barrier, consider the lower barrier (which at low temperatures determines the relaxation rate of a two-level system). At the minimum (to the right) we expand the potential as follows: $U=U_{0}+(\Lambda / 2) x^{2}$ where $x$ is the distance from the minimum. According to statistical mechanics $\left\langle x^{2}\right\rangle=k_{B} T / \Lambda$. If the intersection of the two parabolas is the distance $b$ from the minimum, the estimated lower barrier is given by

$$
\Delta E=\frac{\Lambda}{2} b^{2}=\frac{k_{B} T}{\left\langle x^{2}\right\rangle} \frac{b^{2}}{2} .
$$

Comparing this situation to that of Fig. 1(b), where $b$ is the same, we conclude that 


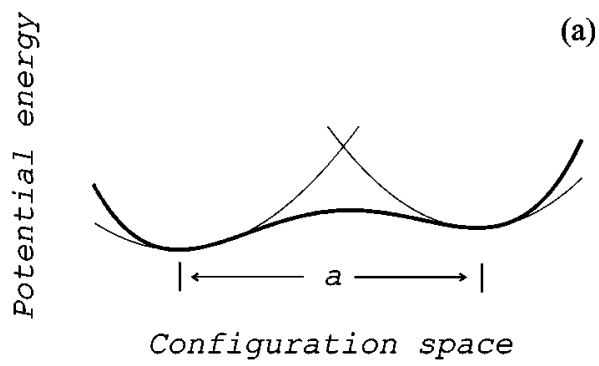

(b)

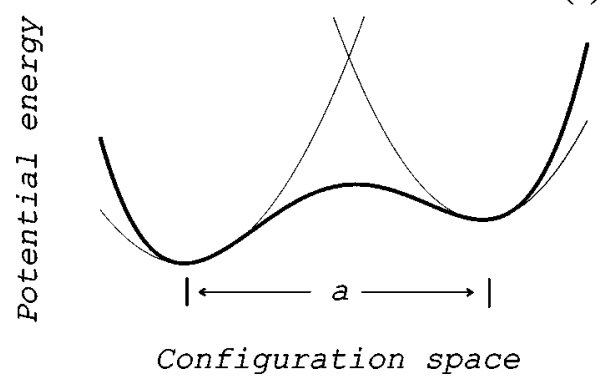

FIG. 1. Comparison of two cases of two potential energy minima with the same distance between, but different curvatures at, the minima. The full curve is the potential energy, while the thin curve gives the potential esitmated by second order Taylor expansions around the minima. According to a classical reasoning used, e.g., in the Marcus theory for electron transfer reactions, the barrier height may be estimated by extrapolating from the minima. The estimated barriers are clearly much larger than the actual barriers; however, going from (a) to (b) the estimated and actual barriers are proportional. This is enough to arrive at Eq. (1).

$$
\Delta E(T) \propto \frac{k_{B} T}{\left\langle x^{2}\right\rangle} .
$$

How does the above reasoning apply to the multidimensional configuration space where the hills and valleys of the energy landscape live? At low temperatures a viscous liquid thermally "populates" only deep minima in configuration space. A transition between two deep minima most likely consists of a whole sequence of transitions between intermediate shallow minima [3]. Nevertheless, there is one or more bottlenecks in this sequence. Our basic assumption is that at different temperatures the bottleneck transitions are the same type of local rearrangements and thus with (virtually) same distance between the two minima in configuration space. They occur, however, as temperature changes in different surroundings, so the minima involved are different and have differing $\left\langle x^{2}\right\rangle$. The relevant mean-square $x$ fluctuation is to be taken in the direction between the minima. The final assumption needed is that this quantity is typical for the minima, i.e., equal to the average over all directions. Consequently, the relevant mean-square $x$ fluctuation may be evaluated simply as the vibrational mean-square fluctuation averaged over all directions. We estimate this quantity by its ensemble average over all minima, $\left\langle x^{2}\right\rangle$. Note that, since the configuration space average $\left\langle x^{2}\right\rangle$ is equal to the single-atom mean-square displacement, this quantity is experimentally accessible in, e.g., incoherent neutron scattering where it enters into the Debye-Waller factor $\exp \left(-Q^{2}\left\langle x^{2}\right\rangle\right)$, where $Q$ is the scattering vector [26].

Recently, Starr, Sastry, Douglas, and Glotzer arrived at Eq. (3) from the free volume perspective [27]. Their work includes a direct numerical confirmation for a glass-forming polymer melt by calculating the free volume $v_{f}$ and subsequently showing, with $v_{f}$ as "mediator," that the temperature dependencies of $\left\langle x^{2}\right\rangle$ and $\tau$ are consistent with Eq. (3).

The next step is to relate $\left\langle x^{2}\right\rangle$ to the instantaneous bulk and shear moduli. On short-time scales a viscous liquid behaves like a solid [14-16,28]. In particular, it has welldefined vibrational eigenstates. We assume that the entire phonon spectrum scales with the long wavelength limit of the phonon dispersion relation. For a one-dimensional solid there is only one elastic constant $C$. The above $\Lambda$ is proportional to $C$ and consequently, $\left\langle x^{2}\right\rangle \propto T / C$. In a threedimensional isotropic solid there are two elastic constants. For each wave vector there are three phonon degrees of freedom, two transverse and one longitudinal. The relevant transverse elastic constant is the isothermal (= adiabatic) shear modulus, while the relevant longitudinal elastic constant is the isothermal ( $\neq$ adiabatic) longitudinal modulus $M$ defined [29] by $M=K+(4 / 3) G$, where $K$ and $G$ are the bulk and shear isothermal moduli. Averaging over the two types of phonons one finds that the vibrational mean-square fluctuation is given by $\left\langle x^{2}\right\rangle \propto T(2 / G+1 / M)$. This applies for a solid. It applies at short times for a viscous liquid as well, if $G$ and $M$ are identified with the liquid's instantaneous isothermal moduli. Thus inserting the expression for $\left\langle x^{2}\right\rangle$ into Eq. (3) we get

$$
\frac{1}{\Delta E(T)} \propto \frac{2}{G_{\infty}(T)}+\frac{1}{M_{\infty}(T)} .
$$

Unfortunately, it is not possible to test Eq. (4) directly because there are no measurements of the instantaneous isothermal bulk modulus.

There are several ways to quantify the temperature variation of $\Delta E(T)$, a liquid's "fragility" [30]. The standard approach utilizes the quantity $m$ introduced by Plazek, Ngai, Böhmer, and Angell [31]: $m=d \log _{10}(\tau) /\left.d\left(T_{g} / T\right)\right|_{T=T_{g}}$, where $T_{g}$ is the calorimetric glass transition temperature defined by $\tau\left(T_{g}\right)=10^{3} \mathrm{~s}$. Simple Arrhenius behavior corresponds to $m=16$; most glass-forming liquids have fragilities between 50 and 150. As an alternative Tarjus and co-workers proposed to measure the degree of non-Arrhenius behavior at any given temperature by the normalized activation energy: $\Delta E(T) / \Delta E(T \rightarrow \infty)$ [12]. It is not obvious a priori, however, that scaling to the high-temperature limit is physically relevant. Inspired by the Grüneisen parameter [26] and Granato's recent work on interstitialcy relations for the viscosity [32] we suggest that a useful unbiased measure of how much the activation energy changes with temperature is its logarithmic derivative, $d \ln \Delta E(T) / d \ln T$. Because the activation energy increases as $T$ decreases, it is convenient to change sign. We thus define the temperature index $I_{\Delta E}$ of the activation energy by

$$
I_{\Delta E}=-\frac{d \ln \Delta E(T)}{d \ln T} .
$$


It is straightforward to show that $m=16\left[1+I_{\Delta E}\left(T_{g}\right)\right]$.

We proceed to express $I_{\Delta E}$ in terms of temperature indices of instantaneous moduli (same definition). First, note that the temperature index $I$ of a sum, $f_{1}+f_{2}$, is a convex combination of the temperature indices $I_{j}$ of $f_{j}: I=\alpha_{1} I_{1}+\alpha_{2} I_{2}$, where $\alpha_{j}=f_{j} /\left(f_{1}+f_{2}\right)$. Equation (4) thus implies

$$
I_{\Delta E}=\frac{2 G_{\infty}^{-1}}{2 G_{\infty}^{-1}+M_{\infty}^{-1}} I_{G_{\infty}}+\frac{M_{\infty}^{-1}}{2 G_{\infty}^{-1}+M_{\infty}^{-1}} I_{M_{\infty}} .
$$

Similarly, $M_{\infty}=K_{\infty}+(4 / 3) G_{\infty}$ implies

$$
I_{M_{\infty}}=\frac{K_{\infty}}{M_{\infty}} I_{K_{\infty}}+\frac{4}{3} \frac{G_{\infty}}{M_{\infty}} I_{G_{\infty}} .
$$

Substituting Eq. (7) into Eq. (6) leads to

$$
I_{\Delta E}=(1-\alpha) I_{G_{\infty}}+\alpha I_{K_{\infty}}
$$

where

$$
\alpha=\frac{M_{\infty}^{-1}}{2 G_{\infty}^{-1}+M_{\infty}^{-1}} \frac{K_{\infty}}{M_{\infty}} .
$$

It is straightforward to show [33] that one always has

$$
\alpha<0.08 \text {. }
$$

Thus more than $92 \%$ of the temperature index of the activation energy derives from the instantaneous shear modulus. The minute influence of the bulk modulus comes about because of the following three factors:

(1) There are two transverse phonon degrees of freedom, but only one longitudinal.

(2) Longitudinal phonons are associated with a larger elastic constant than transverse phonons and thus give less than one-third contribution to the activation energy [Eq. (4)].

(3) $G_{\infty}$ affects also the longitudinal phonons.
We conclude that simplifying [34], but not unreasonable, assumptions in the landscape approach lead to a prediction for the non-Arrhenius behavior which in practice is going to be hard to distinguish from that of the shoving model. The really interesting question is what is the relation between the physics of the two approaches? At first sight they seem quite different. There are, however, similarities leading us to conclude that the physics are actually closely related: The first shoving model assumption (the main contribution to the activation energy is elastic energy) is equivalent to the landscape assumption of a potential where the curvatures at the minima determine the activation energy. The second shoving model assumption (the elastic energy is in the surroundings of the reorienting molecules) is consistent with the landscape assumption that the vibrational mean-square fluctuation is typical, because vibrational eigenstates in an isotropic solid involve all atoms. And the final shoving model assumption (the elastic energy is mainly shear energy) is consistent with Eq. (10). Finally, we would like to remind that the original shoving model derivation of Eq. (2) assumed spherical symmetry [13]. In more realistic scenarios there must be some volume change in the surroundings of the reorienting molecules and thus some contribution to the activation energy from the instantaneous bulk modulus. While it is difficult to give absolute bounds on the magnitude of the bulk contribution, it is noteworthy that Granato-supported by many others (see Ref. [35], and its references) - finds that for defect creation in a crystal the work is overwhelmingly that of a shear deformation. In our view there is no reason why this should not apply for amorphous structure as well.

The reasonings of Ref. [35] and this paper introduce a new theme into condensed matter physics which may be referred to as shear dominance, according to which when both bulk and shear modulus appear in a problem the shear modulus usually plays a much more important role than the bulk modulus.

This work was supported by the Danish Natural Science Research Council.
[1] M. Goldstein, in Modern Aspects of the Vitreous State, edited by J. D. Mackenzie (Butterworths Scientific, London, 1964), p. 90.

[2] G. Harrison, The Dynamic Properties of Supercooled Liquids (Academic, New York, 1976).

[3] S. Brawer, Relaxation in Viscous Liquids and Glasses (American Ceramic Society, Columbus, OH, 1985).

[4] C.A. Angell, J. Non-Cryst. Solids 131-133, 13 (1991).

[5] S.V. Nemilov, Thermodynamic and Kinetic Aspects of the Vitreous State (CRC, Boca Raton, FL, 1995).

[6] P.G. Debenedetti, Metastable Liquids: Concepts and Principles (Princeton University, Princeton, 1996).

[7] J.C. Dyre, J. Non-Cryst. Solids 235-237, 142 (1998).

[8] C.A. Angell, K.L. Ngai, G.B. McKenna, P.F. McMillan, and S.W. Martin, J. Appl. Phys. 88, 3113 (2000).

[9] E. Donth, The Glass Transition (Springer, Berlin, 2001).

[10] In the present paper, for simplicity we shall not distinguish between activation energy and the more correct activation free energy.

[11] J.C. Dyre, Phys. Rev. B 51, 12276 (1995).

[12] D. Kivelson, G. Tarjus, X.-L. Zhao, and S.A. Kivelson, Phys. Rev. E 53, 751 (1996).

[13] J.C. Dyre, N.B. Olsen, and T. Christensen, Phys. Rev. B 53, 2171 (1996).

[14] W. Kauzmann, Chem. Rev. (Washington, D.C.) 43, 219 (1948).

[15] M. Goldstein, J. Chem. Phys. 51, 3728 (1969).

[16] A. Heuer, Phys. Rev. Lett. 78, 4051 (1997); S. Sastry, P.G. Debenedetti, and F.H. Stillinger, Nature (London) 393, 554 (1998); F. Demichemelis, G. Viliani, and G. Ruocco, Phys. Chem. Comm. 9/01889A (1999); T.B. Schrøder, S. Sastry, J.C. Dyre, and S.C. Glotzer, J. Chem. Phys. 112, 9834 (2000); L. Angelani, R. Di Leonardo, G. Ruocco, A. Scala, and F. Sciortino, Phys. Rev. Lett. 85, 5356 (2000); K. Broderix, K.K. Bhattacharya, A. Cavagna, A. Zippelius, and I. Giardina, ibid. 85, 5360 (2000). 
[17] G. Wyllie, Phys. Rep. 61, 329 (1980).

[18] The first derivation of Eq. (2) (for viscosity $\eta$ ) seems to be by Tobolsky, Powell, and Eyring in a paper from 1943 entitled "Elastic-viscous properties of matter" [A. Tobolsky, R.E. Powell, and H. Eyring, in Frontiers in Chemistry, edited by R.E. Burk and O. Grummit (Interscience, New York, 1943), Vol. 1, p. 125]; a brief account of their reasoning is given below [24]. Nemilov proposed Eq. (2) in 1968 from the observed proportionality between glass transition temperature and glass shear modulus (S.V. Nemilov, Zh. Fiz. Khim. 42, 1391 (1968) [J. Phys. Chem. 42, 726 (1968)]) and justified Eq. (2) by arguments combining Eyring's expression for the viscosity with Dushman's prefactor for the rate (barrier energy divided by Planck's constant, not Eyring's prefactor $k_{B} T / h$ ) and the Maxwell relation $\tau=\eta / G_{\infty}$ [5].

[19] N.B. Olsen, J.C. Dyre, and T. Christensen, Phys. Rev. Lett. 81, 1031 (1998).

[20] R.F. Grote and J.T. Hynes, J. Chem. Phys. 73, 2715 (1980); H.-X. Zhou and R. Zwanzig, J. Phys. Chem. A 106, 7562 (2002).

[21] R.A. Marcus, Annu. Rev. Phys. Chem. 15, 155 (1964).

[22] D.F. Calef and P.G. Wolynes, J. Chem. Phys. 78, 470 (1983).

[23] R.W. Hall and P.G. Wolynes, J. Chem. Phys. 86, 2943 (1987); see also C.P. Flynn, Phys. Rev. 171, 682 (1968).

[24] Interestingly, the derivation of Eq. (2) from 1943 by Tobolsky, Powell, and Eyring [18] is closely related to the Hall-Wolynes argument. Tobolsky and co-workers assumed a cosine potential for the molecule in question, which couples linearly to the shear stress field. Thus the barrier is determined by the curvature of the potential at the minimum, which in turn is related to the shear elastic constant. A critique of this model was given by Alfrey and Mooney on the grounds that "the jumping of an individual molecule in a particular direction could be favored by a force field, but not by any stress field"' [T. Alfrey, Jr., The Mechanical Behavior of High Polymers (Interscience, New York, 1948); see also M. Mooney, Trans. Soc. Rheol. 1, 63 (1957)].

[25] After Hall and Wolynes several authors discussed the relevance of $\left\langle x^{2}\right\rangle$ for the average relaxation time of viscous liq- uids, see, e.g, U. Buchenau and R. Zorn, Europhys. Lett. 18, 523 (1992); A.P. Sokolov, A. Kisliuk, D. Quitmann, A. Kudlik, and E. Rössler, J. Non-Cryst. Solids 172-174, 138 (1994); C.A. Angell, Science 267, 1924 (1995); V.N. Novikov, E. Rössler, V.K. Malinovsky, and N.V. Surovtsev, Europhys. Lett. 35, 289 (1996); C.M. Roland and K.L. Ngai, J. Chem. Phys. 104, 2967 (1996); D. Quitmann and M. Soltwisch, Philos. Mag. B 77, 287 (1998); M.M. Teeter, A. Yamano, B. Stec, and U. Mohanty, Proc. Natl. Acad. Sci. U.S.A. 98, 11242 (2001).

[26] C. Kittel, Introduction to Solid State Physics, 7th ed. (Wiley, New York, 1996).

[27] F.W. Starr, S. Sastry, J.F. Douglas, and S.C. Glotzer, Phys. Rev. Lett. 89, 125501 (2002).

[28] J.C. Dyre, Phys. Rev. E 59, 2458 (1999).

[29] L.D. Landau and E.M. Lifshitz, Theory of Elasticity, 2nd ed. (Pergamon, Oxford, 1970).

[30] C.A. Angell, in Relaxations in Complex Systems, edited by K.L. Ngai and G.B. Wright (U.S. GPO Washington, DC, 1985), p. 3.

[31] D.J. Plazek and K.L. Ngai, Macromolecules 24, 1222 (1991); R. Böhmer and C.A. Angell, Phys. Rev. B 45, 10091 (1992); R. Böhmer, K.L. Ngai, C.A. Angell, and D.J. Plazek, J. Chem. Phys. 99, 4201 (1993).

[32] A.V. Granato, J. Non-Cryst. Solids 307-310, 376 (2002).

[33] In terms of $k \equiv K_{\infty} / G_{\infty}$ one has $\alpha(k)=k /[(k+4 / 3)(2 k$ $+11 / 3)]$. This function has its maximum at $k=\sqrt{22} / 3$, where $\alpha=0.0794$.

[34] The assumption that the entire phonon spectrum scales with the macroscopic elastic constants is nontrivial, in particular, because of the large number of short wavelength phonons. The individual contribution from long wavelength phonons to $\left\langle x^{2}\right\rangle$, however, goes as $k^{-2}$ which compensates for their small number $\left(\propto k^{2}\right)$. Nevertheless, if the short wavelength phonon contribution to $\left\langle x^{2}\right\rangle$ does not scale with the macroscopic elastic constants, the derivation does not apply. Moreover, in a real viscous liquid there are localized phonons and our reasoning depends on these either contributing little to $\left\langle x^{2}\right\rangle$ or scaling with the macroscopic elastic constants.

[35] A.V. Granato, Phys. Rev. Lett. 68, 974 (1992). 